\title{
In Vivo Pharmacokinetic and Hemocompatible Evaluation of Lyophilization Induced Nifedipine Solid-Lipid Nanoparticle
}

\author{
Ranjan Kumar Barman1,2, Yasunori Iwao1, Md. Rafiqul Islam³, Yuka Funakoshi', \\ Shuji Noguchi1, Mir Imam Ibne Wahed ${ }^{2}$, Shigeru Itai ${ }^{*}$ \\ ${ }^{1}$ Department of Pharmaceutical Engineering and Drug Delivery Science, Graduate School of Pharmaceutical \\ Sciences, University of Shizuoka, Shizuoka, Japan \\ ${ }^{2}$ Department of Pharmacy, Faculty of Science, University of Rajshahi, Rajshahi, Bangladesh \\ ${ }^{3}$ Department of Pharmacy, Faculty of Biological Science and Technology, Jessore University of Science and \\ Technology, Jessore, Bangladesh \\ Email: ${ }^{*}$ s-itai@u-shizuoka-ken.ac.jp
}

Received 27 February 2014; revised 5 April 2014; accepted 25 April 2014

Copyright (C) 2014 by authors and Scientific Research Publishing Inc.

This work is licensed under the Creative Commons Attribution International License (CC BY). http://creativecommons.org/licenses/by/4.0/

\section{Abstract}

Nifedipine-solid-lipid nanoparticles lyophilized with trehalose (NI-SLN-Tre) were prepared by the high pressure homogenization of a roll mixture consisting of NI and hydrogenated soybean phosphatidylcholine and dipalmitoylphosphatidylglycerol, and in vivo pharmacokinetic properties and their hemocompatibility were determined and compared with those of a NI-SLN suspension. The resulting pharmacokinetic data demonstrated that although no significant differences were observed between the time of peak concentration $\left(T_{\max }\right)$, peak plasma concentration $\left(C_{\max }\right)$, and the area under the curve $\left(A U C_{0 \rightarrow \infty}\right)$ values of both administrated samples, NI tended to be absorbed to a much greater extent from the lyophilized NI-SLN-Tre suspensions because of the enhanced solvation of NI-SLN in gastrointestinal fluid, derived from formation of hydrogen bonds between the polar head groups of the lipids and the 0 -H groups of trehalose. Furthermore, the results of a hemolysis assay revealed that the NI-SLN and NI-SLN-Tre suspensions showed good hemocompatibility properties with hemolysis values of less than $5 \%$. Taken together, the results of this study demonstrate that NI-SLN-Tre exhibits suitable pharmacokinetic properties and good biocompatibility.

\footnotetext{
${ }^{*}$ Corresponding author.
} 


\section{Keywords}

\section{Nifedipine, Solid-Lipid Nanoparticles, Lyophilization, Pharmacokinetics, Hemocompatibility}

\section{Introduction}

Solid-lipid nanoparticles (SLNs) are colloidal carriers made up of lipids that remain solid at both room and body temperature, and offer unique properties such as small particles sizes $(50-500 \mathrm{~nm})$ and much lower and/or higher zeta potentials. SLNs have attracted increasing levels of interest from researchers working in the pharmaceutical sciences in terms of their potential application as alternative drug delivery systems, because they possess good tolerability and stability characteristics, as well as being amenable to scale-up, and allowing for the incorporation of hydrophobic/hydrophilic drugs [1]-[3]. The incorporation of poorly water soluble drugs into SLNs can enhance their gastrointestinal (GI) solubility properties, as well as their absorption and bioavailability [4] [5]. Pandey et al. [6] demonstrated that five oral doses of an antitubercular drug-loaded SLN administered every 10 days provided an equivalent therapeutic benefit to 46 daily doses of the orally administered free drugs.

In a series of recent publications, we described our novel preparation of an SLN suspension of nifedipine (NI-SLN) from two phospholipid carriers [i.e., hydrogenated soybean phosphatidylcholine (HSPC) and dipalmitoylphosphatidylglycerol (DPPG)] using both co-grinding and high pressure homogenization techniques [7]-[10]. This SLN suspension did not show any distinct particle aggregation issues when it was held for up to 4 months at $4^{\circ} \mathrm{C}$ as a long term stability assessment [7] [8]. Furthermore, the application of a lyophilization technique involving the use of trehalose as a cryoprotectant at a concentration of $2.5 \% \mathrm{w} / \mathrm{v}$ led to further increases in the stability of the NI-SLNs in terms of their dissolution pattern, particle size and zeta potential properties, which remained unchanged at $30^{\circ} \mathrm{C}$ and $65 \% \mathrm{RH}$ for up to 6 months [9], suggesting that it is expected that the lyophilized NI-SLNs will also have a prolonged shelf life under ambient conditions. It is noteworthy, that the oral administration of our NI-SLN to rats led to a 4-fold increase in the peak plasma concentration $\left(C_{\max }\right)$ of NI, as well as improved biological half-life and area under the curve $\left(A U C_{0 \rightarrow \infty}\right)$ properties relative to those of a suspension of only NI at an equivalent dose [10]. A variety of different mechanisms have been proposed to account for the passage of nanoparticles through the intestinal mucosa, with most of the current evidence suggesting that particle size has a dramatic impact on the extent of absorption [11] [12]. Saez et al. [13] reported a comparison of the pharmacokinetic properties of polycaprolactone (PCL) nanoparticles immediately after their preparation (100 nm) by nano-precipitation method and after they had been lyophilized $(160 \mathrm{~nm})$. The results of this particular study revealed that the $160 \mathrm{~nm}$ nanoparticles induced a larger first-order mean residence time (MRT) and higher levels of the drug in hepatic circulation, which suggested that even small changes in the particle size of the NPs induced by lyophilization could have a significant effect on the pharmacokinetic properties of the NPs, and that changes resulting from lyophilization should therefore be carefully evaluated. Based on these results and to complement our current understanding of the pharmacokinetic characteristics of the NI-SLN suspension [10], there is an urgent need to evaluate the pharmacokinetic properties of these SLNs following their lyophilization with trehalose (NI-SLN-Tre).

The blood compatibility of nanoparticles is a significant criterion for biomedical and pharmaceutical applications [14]. Given that it may be necessary to administer these agents via intravenous or intramuscular injection to achieve maximum bioavailability, it will be necessary to measure the hemolytic potential of NI-SLN-Tre in blood to develop a better understanding of its blood compatibility. To the best of our knowledge, there have been no reports in the literature to date pertaining to the hemolytic effects of NI-SLNs on the membrane of mammalian erythrocytes, and the evaluation of the hemocompatibility of the NI-SLN-Tre using a hemolysis assay is therefore important.

Hence, the objectives of the current study are to compare the in vivo pharmacokinetic properties of highly stable lyophilized NI-SLN-Tre with those of NI-SLN suspensions and to evaluate the hemocompatibilities of the SLN suspensions and the SLNs lyophilized with trehalose.

\section{Experimental}

\subsection{Materials}

Hydrogenated soybean phosphatidylcholine (HSPC, COATSOME ${ }^{\circledR} \mathrm{NC}-21$ ) and dipalmitoylphosphatidyl-gly- 
cerol (DPPG, COATSOME ${ }^{\circledR}$ MG-6060LS) were purchased from Nippon Oil and Fats Co., Ltd. (Tokyo, Japan). Nifedipine (JPXIV, NI) was provided by Nippon Fine Chemical Co., Ltd. (Osaka, Japan). Trehalose was purchased from Wako Pure Chemical Industries, Ltd. (Osaka, Japan). The membrane filters (pore size: $0.2 \mu \mathrm{m}$ ) were purchased from Toyo Roshi Kaisha Ltd. (Tokyo, Japan). All of the reagents used in the current study were purchased as the highest grades commercially available, and all solutions were prepared using de-ionized distilled water.

\subsection{Preparation of NI-Loaded Solid-Lipid Nanoparticle (NI-SLN) Suspensions and Lyophilized NI-SLNs with Trehalose (NI-SLN-Tre)}

NI-SLN suspensions and the NI-SLNs lyophilized with trehalose were prepared in accordance with the methods reported in our previous papers [9] [10]. Briefly, to prepare the NI-SLNs, a mixture of $40 \mathrm{mg}$ of NI and $1000 \mathrm{mg}$ of lipids (HSPC:DPPG = 5:1 molar ratio) was physically mixed in a mortar for $5 \mathrm{~min}$, and the resulting mixture was co-ground with an R3-IR roll mill (Kodaira Seisakusho Co., Ltd., Tokyo, Japan) for 5 min. The resulting roll mixture was dispersed in $200 \mathrm{ml}$ of de-ionized distilled water, and premixed using a Speed Stabilizer (Kinematica Co., Luzern, Switzerland) at $9000 \mathrm{rpm}$ for $10 \mathrm{~min}$ followed by a high pressure homogenization (Microfluidizer $^{\circledR}$, M110-E/H; Microfluidics, Co., Newton, MA, USA) at 175 MPa with the pass cycle of 100 . The resulting suspension was filtered through a $0.2 \mu \mathrm{m}$ membrane filter and stored in a refrigerator at $4^{\circ} \mathrm{C}$ for further use.

To prepare the NI-SLN-Tre material, $2 \mathrm{ml}$ of the SLN suspension prepared above was diluted to $4 \mathrm{ml}$ with distilled water and added to a vial containing $25 \mathrm{mg}$ of trehalose and mixed well. The vial was then frozen at $-40^{\circ} \mathrm{C}$ for $3 \mathrm{~h}$ and then freeze-dried in a glass chamber for $24 \mathrm{~h}$ using a vacuum pump equipped with a vapor condenser $\left(-20^{\circ} \mathrm{C}, 0.0225\right.$ Torr). The lyophilized material was then subjected to a secondary drying step, where it was dried at $20^{\circ} \mathrm{C}$ for $24 \mathrm{~h}$.

The mean particle size and zeta potential properties of the NI-SLN and rehydrated NI-SLN-Tre suspensions were evaluated using an electrophoretic light scattering (ELS) photometer (Zetasizer Nano ZS, Malvern, Worcestershire, UK). The NI content of each SLN suspension was measured in accordance with the technique used in our previous study [9] using reversed phase HPLC (Shimadzu, Kyoto, Japan) with a reversed phase Cadenza 5CD-C18 column $\left(150 \times 4 \mathrm{~mm}\right.$, i.d.; Imtakt) at $40^{\circ} \mathrm{C}$. HPLC analyses were conducted in the binary mode (dual pump) with ultraviolet (UV) detection at a wavelength of $236 \mathrm{~nm}$. The mobile phase consisted of a 3:2 ( $\mathrm{v} / \mathrm{v})$ mixture of methanol and aqueous formic acid $(0.2 \% \mathrm{v} / \mathrm{v})$, which was delivered in an isocratic manner at a flow rate of $0.4 \mathrm{ml} / \mathrm{min}$. Under these conditions, the retention time of NI was found to be $6.1 \pm 0.1 \mathrm{~min}$.

\subsection{Pharmacokinetics}

\subsubsection{Animals}

Male Sprague-Dawley rats (weight: 280 - 320 g; age: 9 - 11 weeks; Japan SLC, Shizuoka, Japan or Japan Charles River Laboratories, Kanagawa, Japan) were fasted overnight prior to the experiment. All of the procedures used in the current study were conducted in accordance with the guidelines approved by the Institutional Animal Care and Ethical Committee of the University of Shizuoka.

\subsubsection{Pharmacokinetic Experiments}

The NI-SLN and reconstituted NI-SLN-Tre suspensions were both orally administered to the rats at a dose corresponding to $2 \mathrm{mg} / \mathrm{kg}$ of NI. Following the administration of these materials, blood samples were collected via the tail vein at time interval of 1, 5, 10, 30, 60, 120, 240 and $480 \mathrm{~min}$ in Eppendorf tubes containing the anticoagulant heparin. The blood samples were then centrifuged at $4000 \times \mathrm{g}$ for $10 \mathrm{~min}$ to obtain plasma. A sample $(100 \mu \mathrm{l})$ of the plasma was collected and mixed with $500 \mu \mathrm{l}$ of methanol, and the resulting mixture was vortexed for 2 min before being sonicated for $2 \mathrm{~min}$. The material was then centrifuged at $3000 \times \mathrm{g}$ for $10 \mathrm{~min}$ and the supernatant was collected before filtered through a $0.2 \mu \mathrm{m}$ membrane filter. The filtrate was then analyzed by LC-MS/MS to determine the concentration of NI in the plasma. The main pharmacokinetic parameters, including $A U C_{0 \rightarrow \infty}, C_{\max }$, time of peak concentration $\left(T_{\max }\right)$, halflife $\left(t_{1 / 2}\right)$, elimination rate constant $\left(k_{e}\right)$ and MRT were analyzed using the Win Nonlin Pharmacokinetic program.

\subsection{Hemolysis Assay}

The hemocompatibilities of the NI-SLN and reconsti tuted NI-SLN-Tre suspensions were evaluated using a he- 
molysis assay [15]. Erythrocytes were isolated from $2 \mathrm{ml}$ of fresh rabbit blood, and diluted with normal saline to an erythrocyte dispersion of $2 \%$. Different volumes of both samples containing $145 \mu \mathrm{g} / \mathrm{ml}$ of NI $(0.1,0.2,0.3$, 0.4 and $0.5 \mathrm{ml}$ ) were added to five tubes together with $2.5 \mathrm{ml}$ of the $2 \%$ erythrocyte dispersion. Normal saline was then added to each tube to obtain a final volume of $5 \mathrm{ml}$. Positive and negative controls were prepared by the addition of distilled water $(2.5 \mathrm{ml})$ and normal saline $(2.5 \mathrm{ml})$ to $2.5 \mathrm{ml} 2 \%$ erythrocyte dispersions. The samples were incubated at $37^{\circ} \mathrm{C}$ for $2 \mathrm{~h}$ and then centrifuged at $1500 \mathrm{rpm}$ for $10 \mathrm{~min}$, and the amount of released hemoglobin was measured spectrophotometrically at a wavelength of $545 \mathrm{~nm}$ (UV 1750, LC, Japan). The percentage hemolysis values were calculated as follows:

$$
\text { Hemolysis }(\%)=\frac{\left(A_{s}-A_{n}\right)}{\left(A_{p}-A_{n}\right)} \times 100
$$

where $A_{s}$ is the absorbance of the sample, $A_{n}$ is the absorbance of the negative control and $A_{p}$ is the absorbance of the positive control.

\section{Results and Discussion}

\subsection{Physicochemical Properties of NI-SLN and Reconstituted NI-SLN-Tre Suspensions}

As previously reported [9], a slight increase in particle size was observed in the reconstituted NI-SLN-Tre, although some of the other physicochemical properties, including the polydispersity index (PDI) and zeta potential of the NI-SLN-Tre, remained constant (Table 1), suggesting that the use of only $2.5 \%$ trehalose exerted an acceptable lyoprotective effect on the NI-LNPs. This effect was attributed to the membrane-protecting effect of trehalose, which forms hydrogen bonding interactions with the polar head groups of the lipids, as well as disrupting the tetrahedral hydrogen bonding network of water, leading to a reduction in the amount of freezable water.

\subsection{Pharmacokinetic Study}

Figure 1 shows the time courses of NI in the rat plasma samples following the oral administration of the NISLN and reconstituted NI-SLN-Tre suspensions. The pharmacokinetic parameters of these materials are shown in Table 2. Compared with the NI-SLN suspension, the NI-SLN-Tre suspension was slowly and highly absorbed

Table 1. Particle diameter (size), polydispersity index (PDI) and zeta potential (ZP) values of the NI-SLN and reconstituted NI-SLN-Tre suspensions.

\begin{tabular}{cccc}
\hline Sample & Size $(\mathrm{nm})^{\mathrm{a}}$ & PDI $^{\mathrm{a}}$ & ZP $(\mathrm{mV})^{\mathrm{a}}$ \\
\hline NI-SLN suspensions & 68.5 & 0.3 & -62.1 \\
Reconstituted NI-SLN-Tre suspensions & 94.3 & 0.3 & -65.2 \\
\hline${ }^{\mathrm{a}}$ These values are taken from Barman et al. [9]. & &
\end{tabular}

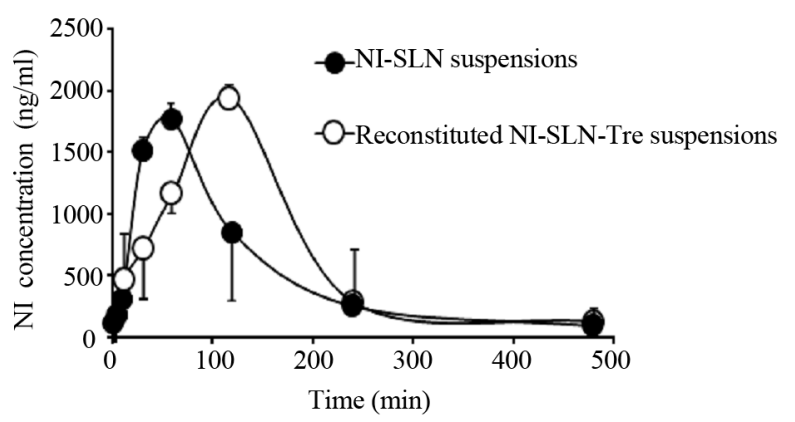

Figure 1. Plasma concentration-time profiles of NI in rats following oral administration of a single dose of $2.0 \mathrm{mg} / \mathrm{kg} \mathrm{NI}$ in the NI-SLN (closed circles) and reconstituted NI-SLN-trehalose (open circles) suspensions. These data are the average values obtained from five experiments ( \pm S.E.). 
Table 2. In vivo pharmacokinetic parameters derived from the kinetic profiles using a noncompartmental approach.

\begin{tabular}{ccc}
\hline Parameters & NI-SLN suspensions & Reconstituted NI-SLN-Tre suspensions \\
\hline$A U C_{0 \rightarrow \infty}\left(\mu \mathrm{g} \cdot \mathrm{min} \cdot \mathrm{ml}^{-1}\right)$ & $238.5 \pm 31.7$ & $273.4 \pm 16.3$ \\
$C_{\max }\left(\mathrm{ng} \cdot \mathrm{ml}^{-1}\right)$ & $1429.4 \pm 341.4$ & $1673.3 \pm 269.4$ \\
$T_{\max }(\mathrm{min})$ & $80.0 \pm 20.0$ & $100.0 \pm 20.0$ \\
$t_{1 / 2}(\mathrm{~min})$ & $96.9 \pm 13.6$ & $76.5 \pm 6.8$ \\
$k_{e}\left(\mathrm{~min}^{-1}\right)$ & $0.0076 \pm 0.0012$ & $0.0118 \pm 0.0035$ \\
MRT (min) & $155.6 \pm 30.3$ & $148.7 \pm 22.6$ \\
\hline
\end{tabular}

These data are the average values obtained from five experiments \pm S.E. AUC: area under the curve; $C_{\max }:$ peak plasma concentration; $T_{\max }$ : time of peak concentration; $t_{1 / 2}$ : half-life; $k_{e}$ : elimination rate constant; MRT: firstorder moment mean residence time.

with $T_{\max }$ and $C_{\max }$ values of $100 \mathrm{~min}$ and $1673.3 \mathrm{ng} \cdot \mathrm{ml}^{-1}$, whereas the NI-SLN suspension gave values of 80 min and $1429.4 \mathrm{ng} \cdot \mathrm{ml}^{-1}$, respectively. A comparison of the $A U C_{0 \rightarrow \infty}$ values for the NI-SLN-Tre and NI-SLN suspensions revealed values of 273.3 and $238.8 \mathrm{~min} \mu \mathrm{g} \cdot \mathrm{ml}^{-1}$, respectively, indicating that although no significant differences were observed between the $T_{\max }, C_{\max }$ and $A U C_{0 \rightarrow \infty}$ values of these materials, NI tended to be absorbed to a much greater extent from the lyophilized NI-SLN-Tre suspension than it was from the NI-SLN suspension. This difference in the absorption properties of the two suspensions was attributed to the enhanced solvation of the NI-SLN-Tre in the GI fluid, derived from the formation of hydrogen bonding interactions between the polar head groups of the lipid and the O-H groups of the trehalose. Given that the absorption of NI has been postulated to occur following its solvation, it is possible that the NI in the NI-SLN-Tre would require a comparatively longer period of time to go into solution, resulting in the delayed $T_{\max }$ and enhanced $C_{\max }$ values. In contrast, the $t_{1 / 2}$ and $k_{e}$ values of the NI-SLN-Tre were $76.5 \mathrm{~min}$ and $0.0118 \mathrm{~min}^{-1}$, respectively, whereas those of the NI-SLN suspension were $96.9 \mathrm{~min}$ and $0.0076 \mathrm{~min}^{-1}$, respectively, suggesting that the NI in the lyophilized formulation was being eliminated from the plasma much more rapidly than that of the intact suspensions. In fact, since the MRT values of the two materials were almost identical (148.7 and $155.6 \mathrm{~min}$ for NI-SLN-Tre and NI-SLN, respectively), it is possible that these two materials could be subjected to different elimination mechanisms following their absorption from the GI tract.

The results of our previous paper demonstrated that the rapid absorption of NI from NI-SLN suspensions was only observed from suspensions of the intact NI drug, in that the $C_{\max }, A U C_{0 \rightarrow \infty}, T_{\max }$ and $\mathrm{t}_{1 / 2}$ of values of the NI-SLN were found to be significantly higher than those of the corresponding NI suspensions and there was also a 4-fold increase in the absorption of NI from the NI-SLN suspension compared with the NI suspension [10]. This report indicated that the use of an NI-SLN suspension would enhance the absorption process through a reduction in the mean particle size of the NI and two phospholipids. Several mechanisms working either alone or in combination may have contributed to the observed increases in the bioavailability of drugs from SLN formulations, including 1 ) changes in the surface active properties of the phospholipids can augment the absorption of drug compounds through variations in the fluidity of the GI membrane or an increase in the affinity of the lipid particles for the GI membrane; 2) variations in the effective absorption of drugs from the SLN because of the inhibitory effect of the DPPG surfactant towards p-glycoproteins, which would lead to a reduction in the efflux of NI from the intestinal membrane; 3) promotion of the dissolution characteristics of these nanomaterials because of their enormous effective surface areas, which would promote the dissolution characteristics of the drug, leading to a higher concentration gradient and an increased rate of absorption; 4) the small sizes of these SLN materials would allow them to adhere more strongly to the GI tract as well entering into the intervillar spaces, which would lead to an increase in the residence time for increased bioavailability; 5) the presence of the lipids would favor lymphatic transport; and 6) the influence of lipids on the preferential uptake of lipid particles by Peyer's patches would also result in an increase in the bioavailability of NI by avoiding clearance through resulting from first pass metabolism. Unfortunately, although the mechanisms involved in the observed increases in the bioavailability of NI from the NI-SLN and NI-SLN-Tre suspensions remain unclear, it is envisaged on the basis of these results that the SLN offers many potential advantages as a suitable carrier for the improved oral bioavailability of NI even after they had been subjected to lyophilization. 


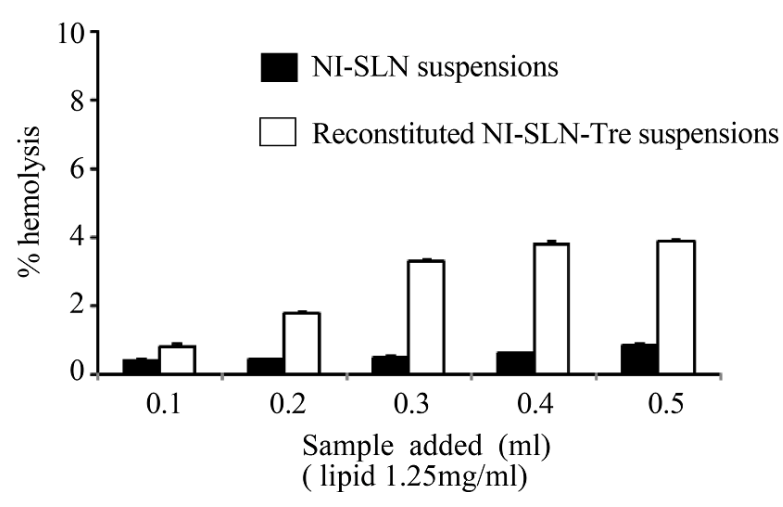

Figure 2. Hemocompatibility study; percentage he-
molysis values of the NI-SLN (closed bars) and re-
constituted NI-SLN-Tre (open bars) suspensions.
These data are the average values obtained from five
experiments ( \pm S.E.).

\subsection{Hemolysis Assay}

In the present study, the hemocompatibilities of the NI-SLN and NI-SLN-Tre suspensions were evaluated using an in vitro hemolysis assay. The results (Figure 2) revealed the effects of different amounts of both samples on the erythrocyte or total blood hemoglobin levels. When sample volumes of $0.1,0.2,0.3,0.4$ and $0.5 \mathrm{ml}$ were added to the assay, the percentage hemolysis values for the NI-SLN suspension were $0.39,0.43,0.49,0.60$, and $0.85 \%$, respectively, indicating a high level of hemocompatibility. In addition, the percentage hemolysis values following the addition of the reconstituted NI-SLN-Tre suspension at the same lipid concentration were found to be less than $5 \%$ for each sample. Values of less than $5 \%$ have been reported to be safe for erythrocytes [15], suggesting that the NI-SLN-Tre suspension also showed a moderate level of hemocompatibility with blood cells. Taken together, these results therefore suggested that both the NI-SLN and lyophilized NI-SLN-Tre suspensions would show good biocompatibility.

\section{Conclusion}

In conclusion, we have developed nifedipine loaded solid-lipid nanoparticles lyophilized with trehalose (NISLN-Tre) that showed a good pharmacokinetic profile, as well as NI-SLN suspension. The $C_{\max }, A U C_{0 \rightarrow \infty}$ and $t_{1 / 2}$ values of the NI-SLN-Tre were found to be almost identical to those of the NI-SLN, expect that the NI tended to be absorbed to a much greater extent from the lyophilized NI-SLN-Tre suspension than it was from the NI-SLN suspension because of the formation of hydrogen bonding interactions between the polar head groups of the lipids and the O-H groups of trehalose. These interactions resulted in the enhanced solvation of the NI-SLN-Tre in the GI fluid, leading to the observed increases in the absorption, bioavailability and residence time properties of the NI in the plasma. These results therefore demonstrated that the lyophilization of NI-SLN with trehalose did not have an adverse impact on the pharmacokinetic properties of the SLNs. In light of the potential therapeutic applications of these materials, we performed a hemolysis assay, which revealed that the NI-SLN and NI-SLN-Tre suspensions were stable towards hemolysis and suggested that these materials were safe for use in the erythrocyte membranes of mammals. Taken together, the results of the current study suggest that lyophilized solid-lipid nanoparticles loaded with potent calcium channel blockers such as NI could play a significant role in the treatment of hypertension and angina pectoris, and that these delivery systems might be safe and effective alternative to the commercially available dosage forms currently used in the clinic in the near future.

\section{Acknowledgements}

We are pleased to acknowledge the Japan Society for the Promotion of Science (JSPS) for providing a research grant through the RONPAKU program. We are also grateful to the Department of Pharmacy, Southeast University, Banani, Dhaka-1213, Bangladesh for their support in conducting the hemolysis assays in the Laboratory of Pharmacology. 


\section{References}

[1] Xie, S.Y., Wang, S.L., Zhao, B.K., Han, C., Wang, M. and Zhou, W.Z. (2008) Effect of PLGA as a Polymeric Emulsifer on Preparation of Hydrophilic Protein Loaded Solid Lipid Nanoparticles. Colloids and Surfaces B: Biointerfaces, 67, 199-204. http://dx.doi.org/10.1016/j.colsurfb.2008.08.018

[2] Mehnert, W. and Mäder, K. (2001) Solid Lipid Nanoparticles: Production, Characterization and Applications. Advanced Drug Delivery Reviews, 47, 165-196. http://dx.doi.org/10.1016/S0169-409X(01)00105-3

[3] Müller, R.H., Mäder, K. and Gohla, S. (2000) Solid Lipid Nanoparticles (SLN) for Controlled Drug Delivery-A Review of the State of the Art. European Journal of Pharmaceutics and Biopharmaceutics, 50,161-177. http://dx.doi.org/10.1016/S0939-6411(00)00087-4

[4] Zhang, Z.W., Bu, H.H., Gao, Z.W., Huang, Y., Gao, F. and Li, Y.P. (2010) The Characteristics and Mechanism of Simvastatin Loaded Lipid Nanoparticles to Increase Oral Bioavailability in Rats. International Journal of Pharmaceutics, 394, 147-153. http://dx.doi.org/10.1016/j.ijpharm.2010.04.039

[5] Müller, R.H., Runge, S., Ravelli, V., Mehnert, W., Thunemann, A.F. and Souto, E.B. (2006) Oral Bioavailability of Cyclosporine: Solid Lipid Nanoparticles $\left(\mathrm{SLN}^{\circledR}\right)$ versus Drug Nanocrystals. International Journal of Pharmaceutics, 317, 82-89. http://dx.doi.org/10.1016/j.ijpharm.2006.02.045

[6] Pandey, R., Sharma, S. and Khuller, G.K. (2005) Oral Solid lipid Nanoparticle-Based Antitubercular Chemotherapy. Tuberculosis, 85, 415-420. http://dx.doi.org/10.1016/j.tube.2005.08.009

[7] Kamiya, S., Yamada, M., Kurita, T., Miyagishima, A., Arakawa, M. and Sonobe, T. (2008) Preparation and Stabilization of Nifedipine Lipid Nanoparticles. International Journal of Pharmaceutics, 354, 242-247. http://dx.doi.org/10.1016/j.ijpharm.2007.10.049

[8] Ohshima, H., Miyagishima, A., Kurita, T., Makino, Y., Iwao, Y., Sonobe, T. and Itai, S. (2009) Freeze-Dried Nifedipine-Lipid Nanoparticles with Long-Term Nano-Dispersion Stability after Reconstitution. International Journal of Pharmaceutics, 377, 180-184. http://dx.doi.org/10.1016/j.ijpharm.2009.05.004

[9] Barman, R.K., Iwao, Y., Funakoshi, Y., Ranneh, A.H., Noguchi, S., Wahed, M.I.I. and Itai, S. (2014) Development of Highly Stable Nifedipine Solid-Lipid Nanoparticles. Chemical and Pharmaceutical Bulletin, in Press.

[10] Funakoshi, Y., Iwao, Y., Noguchi, S. and Itai, S. (2013) Lipid Nanoparticles with No Surfactant Improve Oral Absorption Rate of Poorly Water-Soluble Drug. International Journal of Pharmaceutics, 451, 92-94. http://dx.doi.org/10.1016/j.ijpharm.2013.04.050

[11] McClean, S., Prosser, E., Meehan, E., O’Malley, D., Clarke, N., Ramtoola, Z. and Brayden, D. (1998) Binding and Uptake of Biodegradable Poly-DL-Lactide Micro- and Nanoparticles in Intestinal Epithelia. European Journal of Pharmaceutical Sciences, 6, 153-163. http://dx.doi.org/10.1016/S0928-0987(97)10007-0

[12] Jani, P., Halbert, G.W., Langridge, J. and Florence, A.T. (1990) Nanoparticle Uptake by the Rat Gastrointestinal Mucosa: Quantitation and Particle Size Dependency. Journal of Pharmacy and Pharmacology, 42, 821-826. http://dx.doi.org/10.1111/j.2042-7158.1990.tb07033.x

[13] Saez, A., Guzman, M., Molpeceres, J. and Aberturas, M.R. (2000) Freeze-Drying of Polycaprolactone and Poly (D,L-Lactic-Glycolic) Nanoparticles Induce Minor Particle Size Changes Affecting the Oral Pharmacokinetics of Loaded Drugs. European Journal of Pharmaceutics and Biopharmaceutics, 50, 379-387. http://dx.doi.org/10.1016/S0939-6411(00)00125-9

[14] Rejinold, N.S., Muthunarayanan, M., Divyarani, V.V., Sreerekha, P.R., Chennazhi, K.P., Nair, S.V., Tamura, H. and Jayakumar, R.J. (2011) Curcumin-Loaded Biocompatible Thermoresponsive Polymeric Nanoparticles for Cancer Drug Delivery. Journal of Colloid and Interface Science, 360, 39-51. http://dx.doi.org/10.1016/j.jcis.2011.04.006

[15] Huang, X., Chen, Y., Peng, D., Li, Q., Wang, X., Wang, D. and Chen, W. (2013) Solid Lipid Nanoparticles as Delivery Systems for Gambogenic Acid. Colloids and Surfaces B: Biointerfaces, 102, 391-397. http://dx.doi.org/10.1016/j.colsurfb.2012.08.058 